\title{
COMPARATIVE ANALYSIS OF THE PERFORMANCE OF RESOURCE ALLOCATION ALGORITHMS IN LONG TERM EVOLUTION NETWORKS
}

\author{
U. N. Nwawelu', ${ }^{1,}$, C. I. Ani ${ }^{2}$ and M. A. Ahaneku ${ }^{3}$ \\ 1,2,3DePartment of ELECTRONIC ENGINEERING, UNIVERSITY OF NigERIA, NSUKKA, ENUGU STATE, NIGERIA. \\ E-mail addresses: ${ }^{1}$ udodegreat@yahoo.com, ${ }^{2}$ cosmas.ani@unn.edu.ng, ${ }^{3}$ mamilus.ahaneku@unn.edu.ng
}

\begin{abstract}
The growth in the good number of real-time and non-real-time applications has sparked a renewed interest in exploring resource allocation schemes that can be efficient and fair to all the applications in overloaded scenarios. In this paper, the performance of six scheduling algorithms for Long Term Evolution (LTE) downlink networks were analyzed and compared. These algorithms are Proportional Fair (PF), Exponential/Proportional Fair (EXP/PF), Maximum Largest Weighted Delay First (MLWDF), Frame Level Scheduler (FLS), Exponential (EXP) rule and Logarithmic (LOG) rule. The performances of these algorithms were evaluated using an open source simulator (LTE simulator) and compared based on network parameters which include: throughput, delay, Packet Loss Ratio (PLR), and fairness. This work aims at giving insight on the gains made on radio resource scheduling for LTE network and to $x$-ray the issues that require improvement in order to provide better performance to the users. The results of this work show that FLS algorithm outperforms other algorithms in terms of delay, PLR, throughput, and fairness for VoIP and video flow. It was also observed that for Best Effort (BE) flows, FLS outperforms other algorithms in terms of delay and PLR but performed least in terms of throughput and fairness.
\end{abstract}

Keywords: LTE, MLWDF, FLS, PLR, Algorithms, Resource Allocation.

\section{INTRODUCTION}

In recent times, the number of mobile subscribers and the volume of traffic generated by them have heavily increased [1]. This has brought about the introduction of a packet based broadband system referred to as LTE networks. LTE network is deployed to provide a smooth migration towards fourth generation (4G) cellular wireless systems. It can be operated in different spectrum allocations from 1.4 to $20 \mathrm{MHz}[2,3]$. LTE provides high peak data rates up to $300 \mathrm{Mbps}$, improves spectrum efficiency, and reduces radio access delays [3, 4]. The technological breakthrough achieved by LTE network over its predecessors placed the network in the position to handle highly demanding services.

LTE technology uses Orthogonal Frequency Division Multiple Access (OFDMA) and Single Carrier Frequency Division Multiple Access (SC-FDMA) as its radio access technology for downlink and uplink transmission [5]. In downlink transmission, radio resources are arranged in both frequency and time domains and are referred to as resource blocks (RBs) $[3,6]$. In the frequency domain, a RB consists of 12 consecutive subcarriers $(180 \mathrm{KHz}$ total bandwidth) while in the time domain, it is made up of a time slot of 0.5 ms duration $[3,6,7]$. A time slot consists of a number of OFDM symbols which can be either seven (normal cyclic prefix) or six (extended cyclic prefix). The normal cyclic prefix is used in urban cells and high data rate applications while the extended cyclic prefix is used in special cases like multi-cell broadcast and in very large cells (e.g. rural areas, low data rate applications). In LTE, one radio frame has a length of $10 \mathrm{~ms}$. Each radio frame is divided into ten equally sized sub-frames of $1 \mathrm{~ms}$ in length. Each sub-frame consists of two equally sized slots of $0.5 \mathrm{~ms}$ in length. A collection of sub-frames with common modulation and coding schemes are referred to as Transport Blocks (TBs) [3]. The way resource blocks (RBs) are arranged in a sub-frame distinguishes uplink access technique from downlink technique. For downlink, any arbitrary two RBs (in time domain) are assigned to a user; while in the uplink, two contiguous RBs or sub-frame are assigned to a user. This arrangement ensures that the uplink access scheme (SCFDMA) minimizes the high peak average power ratio (PAPR) experience in the downlink access scheme (OFDMA) $[3,8,9,10]$. Scheduling in LTE is performed at every $1 \mathrm{~ms}$ interval referred to as Transmit Time Interval (TTI) $[3,8,9]$. 
Scheduling involves the distribution of scarce radio resources among the active users to satisfy their QoS needs $[8,11,12,13]$. In literature, researches have been done on radio resource allocation in LTE downlink network. Different procedures and decisions have been used to design and test the performance of schedulers. The key design aspects range from complexity, scalability, spectral efficiency, fairness, to QoS provisioning $[14,15]$. Depending on the research goal, schedulers prioritize the users based on criteria such as channel condition, packet delay, service type, resource allocation policies $[11,16,17,18]$. Although, not all the parameters are used at the same time to achieve the set goals. These schedulers have been classified into channel unaware, channel aware/QoS unaware, channel aware/QoS aware schedulers $[8,19,20]$. First in First out (FIFO) [8], Round Robin (RR) [21], Blind Equal Throughput (BET) [22], Weighted Fair Queuing (WFQ) [8], Resource Preemption (RP) [8], and Guaranteed Delay scheme which include Earliest Deadline First (EDF) [23] and Largest Weighted Delay First (LWDF) $[23,24]$ are grouped as channel unaware resource scheduling schemes. Maximum Rate (MR) [8], Proportional Fair (PF) [8], Throughput to Average (TTA) [22], and Buffer-Aware Traffic-Dependent (BATD) [25] are grouped as channel aware/QoS unaware schemes. Finally, channel aware/QoS aware schemes include scheduler for guaranteed data rates and scheduler for guaranteed delay requirement which include MLWDF, Exponential Proportional Fair (EXP-PF), FLS, EXP rule, LOG rule etc.

In this paper, we analyzed and compared the scheduling algorithms such as PF, EXP/PF, MLWDF, FLS, EXP rule and LOG rule. These are some of the popular scheduling algorithms for Long Term Evolution (LTE) downlink network. The comparison was based on throughput, delay, Packet Loss Ratio (PLR), and fairness performance metrics. This work aims at giving insight on the improvement made on radio resource scheduling for LTE network and to $\mathrm{x}$-ray the issues that require improvement in order to provide improved performance to the users. Our major concern is the performance of these algorithms at overloaded conditions.

This paper is organized as follows. Section 2 presented some scheduling schemes and some works that compared their performances. In section 3 , the physical model of a packet scheduler is introduced. In section 4, the methodology used was presented. Results presentation and analysis is given in section 5 . The paper is concluded in section 6 .

\section{REVIEW OF THE RELATED WORKS}

Proportional fair algorithm is the first evolutionary scheduling scheme researchers always use as reference scheduler. It has its clear application in almost all the state-of-the-art schedulers. Authors always use PF to provide a balance between throughput and fairness among the applications. This scheme takes into account both the experienced channel quality and the past user throughput before assigning radio resources to a user. Some of the works that employed PF schedulers are [26, $27,28,29,30,31,32,33,34,35,36]$. The readers are encouraged to go through them for an insight. PF scheduler assigns available timeslots to the user, $i$, with the highest metric. PF metric can be expressed analytically as;

$$
\mathrm{U}_{i, j}^{P F}=\max \left[\frac{\mathrm{r}_{i}(\mathrm{t})}{\mathrm{R}_{i}(\mathrm{t})}\right]
$$

where, $r_{i}(t)$ is the data rate corresponding to the channel state of the user $i$, at time $t . \mathrm{R}_{i}(\mathrm{t})$ is the past average throughput (data rate) experienced by the ith user at time $t$.

Though PF algorithm met scheduling requirements of non real-time services, but is not ideal for the real-time services. This is because the PF scheme did not consider the delay of data packet which is one of the attributes of LTE network.

\subsection{Maximum Largest Weighted Delay First (MLWDF)}

Maximum Largest Weight Delay First is a QoS aware resource allocation scheduling scheme for real time and non real time services. The scheme combined QoS class service differentiation mechanism with a proportional fair scheduling (PF) scheme. This was achieved by extending the PF scheme with packet delay and packet loss dependent components. For MLWDF scheduling scheme, resources are allocated to the user with the maximum metric which is made up of the product of the HOL packet delay of the user $\left(\mathrm{D}_{\text {ног }}\right)$, the channel capacity with respect to flow and the QoS differentiating factor [9, $19,37]$. Thus, the priority metric of MLWDF scheduler can be expressed analytically as follows $[3,8,19,20,37]$.

$$
\mathrm{U}_{\mathrm{i}, \mathrm{j}}^{\mathrm{MLDWF}}=\max \left[\alpha_{\mathrm{i}} \mathrm{D}_{\mathrm{HOL}, \mathrm{i}}(\mathrm{t}) \frac{\mathrm{r}_{\mathrm{i}}(\mathrm{t})}{\mathrm{R}_{\mathrm{i}}(\mathrm{t})}\right] ; 1 \leq \mathrm{i} \leq \mathrm{N}
$$

where $\alpha_{i}=-\frac{\left(\log \delta_{i}\right)}{\tau_{i}}, \mathrm{r}_{i}(\mathrm{t})$ and $\mathrm{R}_{i}(\mathrm{t})$ represent the same terms as stated in PF scheduler $\alpha_{i}$ is the weight parameter; $\tau_{i}$ is the delay threshold of user $i$ s packets, $\delta_{i}$ is the maximum probability for HOL packet delay of the ith user to exceed the delay threshold of ith user. $\mathrm{D}_{\mathrm{HOL}, i}(\mathrm{t})$ is the head of line (HOL) packet delay (time difference between the current time, the arrival time of a packet) of user $i$ at time $t$ and $\mathrm{N}$ is the the number of users.

\subsection{Exponential/Proportional Fairness (EXP/PF) scheme} $\mathrm{EXP} / \mathrm{PF}$ scheme was developed to increase the priority of real time flows over non real time flows. It schedules 
multiples users at each scheduling time due to availability of multiple groups of subcarriers to be shared by all users within the system at the same time [38]. For the real-time services, they receive higher priorities when their HOL packet delays are approaching the delay deadline. EXP/PF takes into consideration the characteristics of an exponential and PF function of the end-to-end delay of the packet to be transmitted. EXP/PF distinguishes between real time and best effort flows. For best effort flows, EXP/PF becomes PF while for real time $\mathrm{EXP} / \mathrm{PF}$ is given as;

$$
\begin{gathered}
\mathrm{U}_{i, j}^{E X P / P F}=\max \left(\alpha_{i} \frac{\mathrm{r}_{i}(\mathrm{t})}{\mathrm{R}_{i}(\mathrm{t})} \exp \left(\frac{\alpha_{i} \mathrm{D}_{H O L, i}(\mathrm{t})-\overline{\mathrm{D}_{H O L, l}(\mathrm{t})}}{1+\sqrt{\overline{\mathrm{D}_{H O L, l}(\mathrm{t})}}}\right)\right) \\
\overline{\mathrm{D}_{H O L, l}(\mathrm{t})}=\frac{1}{\mathrm{~N}} \sum_{i=1}^{\mathrm{N}} \alpha_{i} \mathrm{D}_{H O L, i}(\mathrm{t})
\end{gathered}
$$

All the parameters employed by EXP/PF inherited their meanings from MLWDF scheduler.

\subsection{Frame Level Scheduler (FLS)}

In Frame Level Scheduler (FLS), two level scheduling schemes were designed and two different algorithms were implemented in these two levels [37]. For the upper level, resource allocation based on discrete time linear control theory is implemented. It computes the amount of data that each real-time source should transmit within a single frame, to satisfy its delay constrains [11]. For the lower level, radio resources are assigned to user through a PF scheduler. This was done to ensure a good level of fairness among multimedia flows. The data $\mathrm{U}_{\mathrm{i}}(\mathrm{k})$ to be transmitted during the $k$ th frame can be obtained by passing a signal $\mathrm{q}_{\mathrm{i}}(\mathrm{k})$ through a time-invariant linear filter with pulse response $h_{i}(k)$ and in given as;

$$
\mathrm{u}_{\mathrm{i}}(\mathrm{k})=\mathrm{h}_{\mathrm{i}}(\mathrm{k}) * \mathrm{q}_{\mathrm{i}}(\mathrm{k})
$$

Where $u_{i}(k)$ is the amount of data that is transmitted during the kth frame, * is the discrete time convolution

\subsection{Exponential (EXP) Rule}

EXP rule algorithm was designed with the aim to optimize the throughput $[37,39]$. This scheme selects a single user/queue to receive service in every scheduling instant. It uses the information of the channel and it queue without any prior knowledge of arrival and channel statistics of traffic. Thus, the priority metric of EXP rule scheduler can be expressed analytically as follows;

$$
\mathrm{U}_{i, j}^{\text {EXP rule }}=\max \left(b_{i} \exp \left(\frac{\alpha_{i} \mathrm{D}_{\mathrm{HOL}, i}(\mathrm{t})}{c+\sqrt{\left(\frac{1}{\mathrm{~N}_{\mathrm{rt}}}\right) \sum_{\mathrm{i}} \mathrm{D}_{\mathrm{HOL}, i}}}\right) * \frac{\mathrm{r}_{i}(\mathrm{t})}{\mathrm{R}_{i}(\mathrm{t})}\right)(6)
$$

where, according to $[8,40]$, the optimal parameters set is;

$$
\left\{\begin{array}{c}
\alpha_{i} \in\left[\frac{5}{0.99 \tau_{\mathrm{i}}}, \frac{10}{0.99 \tau_{\mathrm{i}}}\right] \\
b_{i}=\frac{1}{\mathrm{E}\left[\frac{\mathrm{r}_{i}(\mathrm{t})}{\mathrm{R}_{i}(\mathrm{t})}\right]} \\
c=1
\end{array}\right.
$$

\subsection{Logarithmic (LOG) Rule}

LOG rule algorithm balances QoS metrics such as mean delay and robustness. It allocates resources to users in the same manner EXP rule does, the only difference is that the LOG rule has prior knowledge of arrival and channel statistics of traffic. This helps to schedule the user with current highest rate and is referred to as opportunistic scheduling [41]. For the LOG rule the expression is given as [8].

$$
\mathrm{m}_{i j}^{\mathrm{LOGrule}}=\max \left[\mathrm{b}_{i} \log \left(\mathrm{c}+\alpha_{i} \mathrm{D}_{\mathrm{HOL}, i}\right) \frac{\mathrm{r}_{i}(\mathrm{t})}{\mathrm{R}_{i}(\mathrm{t})}\right]
$$

where; $\alpha_{i}, b_{i}$, and $c$ are tunable parameters. Optimal parameters as given in [40] are;

$$
\mathrm{b}_{\mathrm{i}}=\frac{1}{\mathrm{E}\left[\frac{\mathrm{r}_{\mathrm{i}}(\mathrm{t})}{\mathrm{R}_{\mathrm{i}}(\mathrm{t})}\right]}, \mathrm{c}=1.1 \text {, and } \alpha_{i}=\frac{5}{0.99 \tau_{\mathrm{i}}}
$$

In line with the algorithms presented above, authors carried out performance analysis on some of the popular algorithms. Authors in [38] compared the performance of the EXP/PF and M-LWDF algorithms of video streaming and web browsing. The simulation results show that M-LWDF outperforms EXP/PF at lower load while EXP/PF performs better at higher loads. In [42], performance of PF, EXP, and M-LWDF algorithms were evaluated under mixed traffic scenarios. Simulation results show a slight predominance of M-LWDF algorithm over EXP/PF in the case of packet loss and data throughput while PF scheduler is considerably outperformed by the two other schedulers. For the performance of the algorithm studied so far, MLWDF algorithm always achieves better throughput and fairness performance for real time services. This is because, the parameters closely related to real time services such as QoS and HOL packet delay are incorporated in the scheme. But the scheme is not suitable for non real time flows because packets delay does not have a significant role. The performance of FLS, EXP rule and LOG rule algorithm were evaluated for video traffic in LTE network [11]. The results from the simulations shows that FLS scheme outperforms in terms of average system throughput, average packet delay, PLR, and with a satisfactory level of fairness. The closest work to ours is that presented by the authors in $[11,42]$. However, the impact of these algorithms on the throughput and fairness of the BE applications under overloaded conditions still remain silent. In this work, in addition to PF, MLWDF, and EXP/PF scheduler compared 
by authors in [42], we also carried out comparative study on three more schedulers for LTE downlink systems. Beside throughput, packet loss rate, and packets delays, we also analyze fairness and throughput experienced by this application with emphasis on BE applications under overloaded conditions. These are the main contributions of this paper.

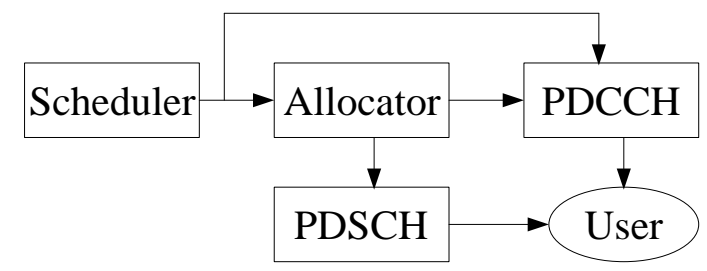

Fig. 1(a): Physical Model of a Downlink Resource Allocation

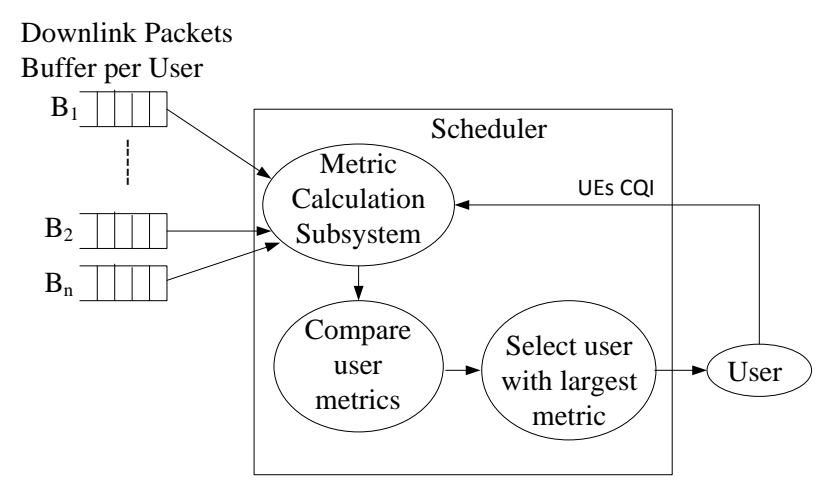

Fig. 1(b): Physical Model of a Downlink Scheduler

Table 1: Simulation Parameters

\begin{tabular}{ll}
\hline Parameters & Value \\
\hline Bandwidth & $10 \mathrm{MHz}$ \\
Carrier frequency & $2 \mathrm{GHz}$ \\
Frame structure & FDD \\
Number of OFDM symbols per slot & 7 \\
Scheduling time (TTI) & $1 \mathrm{~ms}$ \\
SubCarriers per RB & 12 \\
SubCarrier spacing & $15 \mathrm{kHz}$ \\
UE application flow & One video, one VoIP, \\
Cell radius & one BE \\
Maximum delay & $1 \mathrm{Km}$ \\
Simulation duration & $0.1 \mathrm{~s}$ \\
Flow duration & $150 \mathrm{~s}$ \\
Video, VoIP, and BE bit rates & $120 \mathrm{~s}$ \\
\hline
\end{tabular}

\section{PHYSICAL MODEL OF A PACKET SCHEDULER}

For the purpose of this work, emphasis is laid on the scheduling section of the downlink resource allocator. A simplified physical model of a downlink resource allocator and scheduler are shown in Fig. (1a) and (1b). In every TTI, each user sends information via a signaling channel to the scheduler at Enhanced Node Base Station (eNB). The information indicates the channel quality/condition of the user per channel. This information is referred to as the user channel quality indicator (CQI) $[3,12,19]$. This CQI value is chiefly dependent on the signal to interference noise ratio (SINR) experienced by the user for each available channel [3]. At eNB, a buffer is assigned for each user. Packets arriving at the buffer are time stamped and queued for transmission on FIFO basis. On every TTI, the scheduler dynamically selects the user(s) to be allocated resources based on some scheduling parameters such as channel condition, traffic type, Head of Line (HOL) packet delay, queue status $[3,19]$. The user to be selected is the one with highest metric based on the scheduling decision of the scheduler. When the users are scheduled, the allocator allocates the selected user with RBs. The allocated RBs are used to determine the modulation, coding and power schemes. Once the modulation, coding and power schemes are determined, user is notified through the Physical Downlink Control Channel (PDCCH). The user uses this information to decode its packet at the next TTI on the Physical Downlink Shared Channel (PDSCH) $[3,9]$.

\section{METHODOLOGY}

This section explained the research methodology we used to compare the performance of PF, EXP/PF, MLWDF, FLS, EXP rule and LOG rule scheduling schemes.

\subsection{Simulation with LTE-Sim}

Any good network simulation tool should closely reflect the true behavior of a network. Such simulation tool must also be versatile, robust, user friendly and traceable [43]. Because LTE-Sim possesses these properties, we used it for our simulation. LTE-Sim is a discrete time system level simulator that supports codes written in $\mathrm{C}++$ language. The simulator covers several aspects of LTE network such as Evolved Universal Terrestrial Radio Access (E-UTRAN) and the Evolved Packet System (EPS). LTE-Sim supports single and heterogenous multi-cell environments, QoS management, multi user's environment, user mobility, handover procedure, and frequency reuse techniques. Three kinds of networks nodes are modeled in LTE-Sim: user equipment (UE), enhanced node base station (eNB), and Mobility Management Entity/Gateway (MME/GW). At the application layer, the simulator implements four different traffic generators and it has support for the management of data radio bearer. Also AMC scheme and Channel Quality Indicator Feedback have been developed in this simulator $[42,44,45]$. Finally this simulator implemented some of the well known scheduling schemes of which PF, MLWDF, and EXP/PF.

In our simulation approach, users are uniformly distributed in a cell with fixed eNB and they are moving at speed of $3 \mathrm{kmph}$. The eNB is located at the centre of 
the cell and it controls all the available RBs. A number of user equipments (UEs) ranging from 10 to 100 are connected to eNB. Each UE receives one VoIP flow, one video flow and one Best Effort (BE) flow simultaneously. Also, users report its channel condition to eNB. The rest of simulation parameters used for analysis is shown in Table 1. In order to ensure high level of reliability of the results, each simulation time lasted for $150 \mathrm{sec}$ and all simulation results are averaged over five simulations.

\subsection{Performance Metrics}

The performance of any network is the function of the QoS experienced by the user of such network. In our work, throughput, delay, PLR, and fairness performance metrics were used to assess the performance of each algorithm.

\subsubsection{Throughput}

Throughput measures the rate of useful bits successfully transmitted through a network [3]. This is given as;

$$
\text { Throughput }=\frac{\mathrm{P}_{\text {transmit }}}{\mathrm{t}}
$$

where, $\mathrm{P}_{\text {transmit }}$ is the size of the transmitted packets and $t$ is the time it takes to transfer the packets per each user.

\subsubsection{Packet Loss Rate}

PLR measures the percentage of packets of data travelling across a physical channel which could not reach their destination [37]. This was calculated using the relationship:

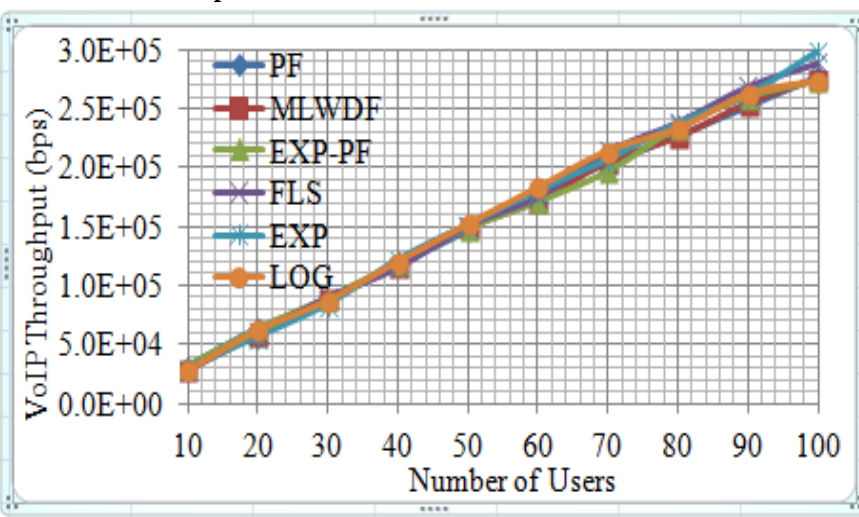

Figure 3: VoIP Throughput for the Six Algorithms

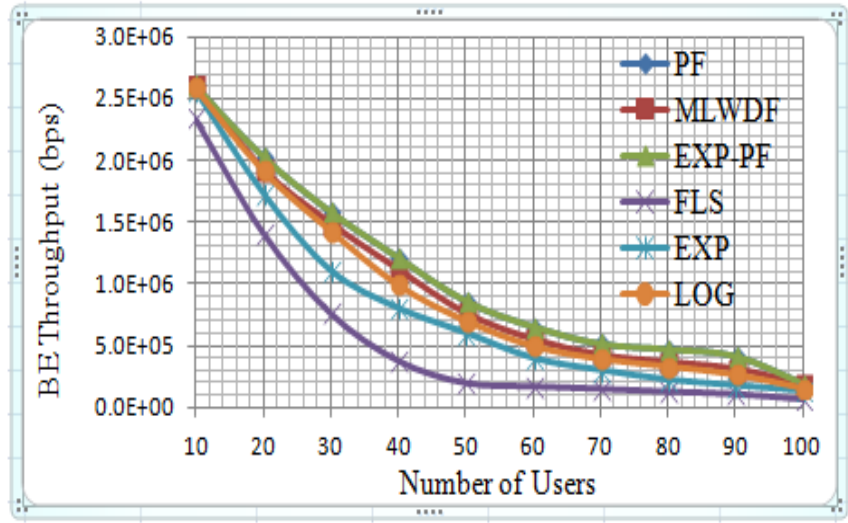

Figure 5: BE Throughput for the Six Algorithms.

Nigerian Journal of Technology

$$
\text { PLR }=\left(\frac{P_{\text {transmit }}-P_{\text {recieve }}}{P_{\text {transmit }}}\right) \times 100
$$

where, $\mathrm{P}_{\text {recieve }}$ is the size of the received packets.

\subsubsection{Delay}

Delay measures the time that elapsed between the time packets departed and the time it got to its destination.

\subsubsection{Fairness Index}

Fairness index is obtained by considering the throughput achieved by each flow at the end of each simulation. Fairness level received by each flow according to Jain's fairness index is given as [46];

$$
\text { Fairness }_{\text {index }}=\frac{\left(\sum \mathrm{x}_{\mathrm{i}}\right)^{2}}{\mathrm{n} \times \sum \mathrm{x}_{\mathrm{i}}^{2}}
$$

where, $\mathrm{x}_{\mathrm{i}}$ is the throughput of the user, $i$, and $\mathrm{n}$ is the number of active flows.

\section{RESULTS ANALYSIS}

The schedulers were simulated to obtain the graphical relationships of figures 3 - 14. Furthermore, the data collected from each scheduler for the 100 users of the eNB were aggregated and presented in percentage. This is done in order to aid statistical comparison.

The throughput for each of the algorithms with number of users for the three different flows (VoIP, video and BE) are shown in Figures 3, 4 and 5. The higher values of throughput indicate better performance of the scheduler.

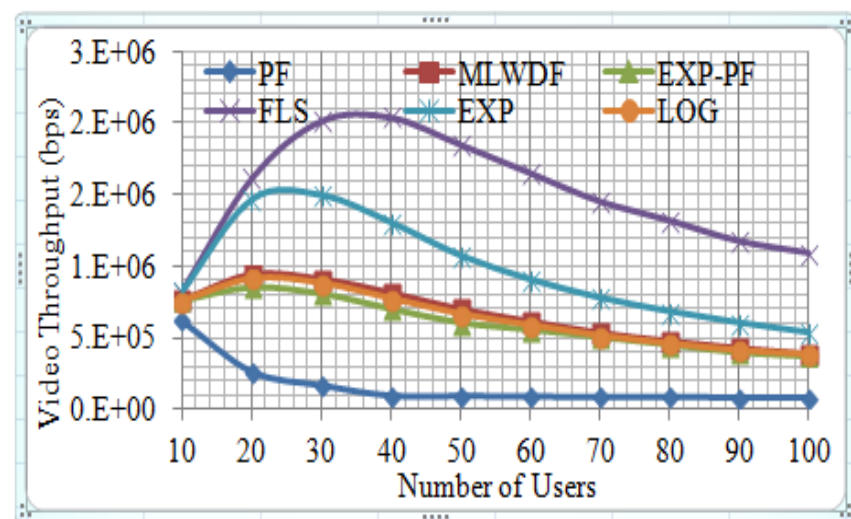

Figure 4: Video Throughput for the Six Algorithms

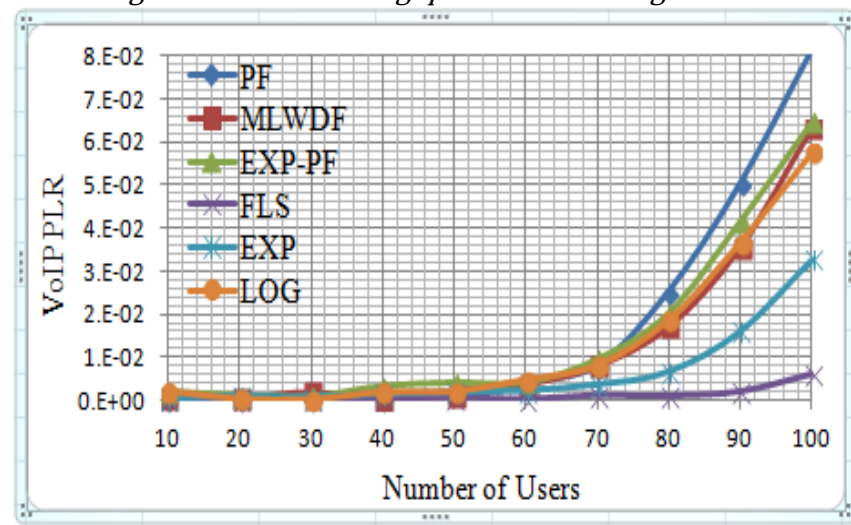

Figure 6: VoIP PLR for the Six Algorithms

Vol. 36, No. 1, January 2017 
In Figure 3, it is observed that the average throughputs for the six algorithms with number of users are quite close with small differences. However, FLS scheduler performed better than the EXP rule scheduler which came second with an average data difference of $0.31 \%$. Figure 4 shows the relationship between the video throughput with the number of users and for the six algorithms. The video throughput increases with the number of users and then decreases. Average data obtained show that the FLS scheduler performed better than the EXP rule, MLWDF, LOG rule, EXP-PF, and PF with an average difference of $14.64 \%, 20.01 \%, 20.1 \%$, $20.22 \%$, and $29.5 \%$ respectively.

Figure 5 shows the plot of throughput for PF, MLWDF, EXP-PF, EXP rule, and LOG rule for the BE flows. The average data collected inferred that, the EXP-PF scheduler performed better than PF, MLWDF, LOG rule, EXP rule, and FLS schedulers with an average difference of $0.01 \%, 1.48 \%, 2.42 \%, 6.1 \%$, and $19.91 \%$ respectively. Since EXP-PF scheduler performed better while FLS scheduler performed least, it shows the FLS scheduler favored real-time services more than non real-time services. The persistent increase in the number of active real time users can lead to the starvation of BE users in overloaded scenarios. A sharp drop in throughput of BE services for the six algorithms; when 100 active users are accessing the network portray this starvation phenomenon.

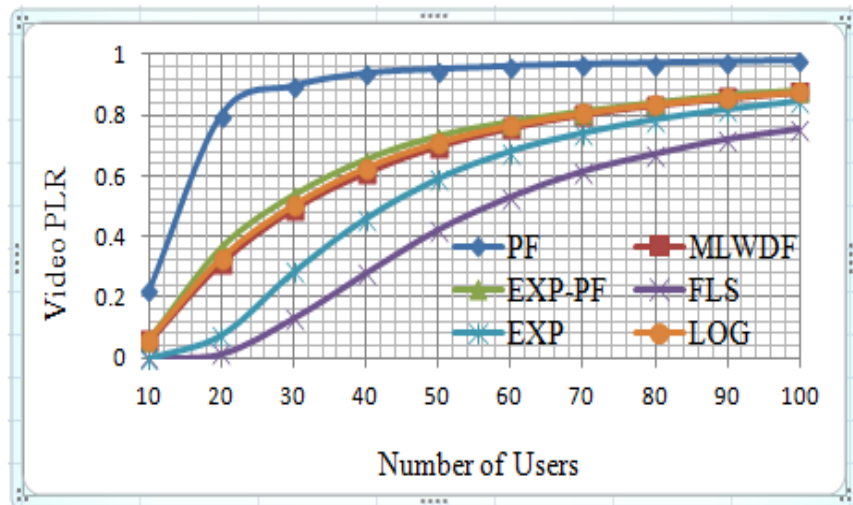

Figure 7: Video PLR for the Six Algorithms

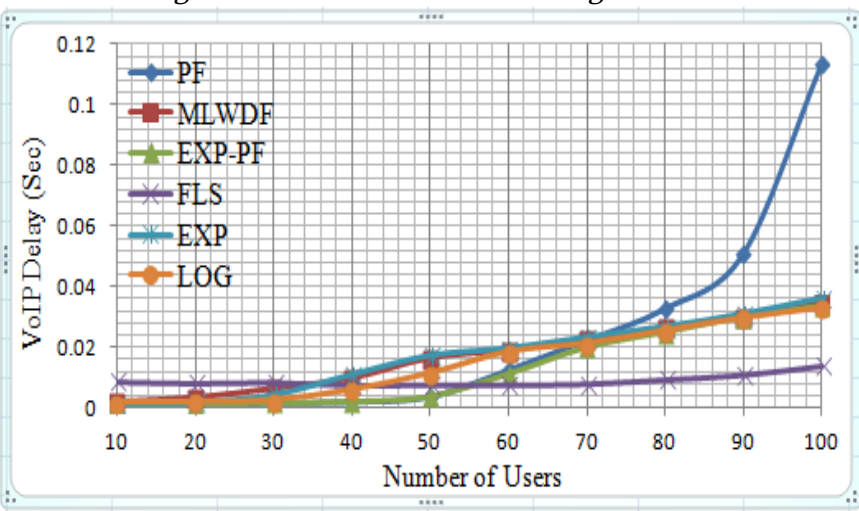

Figure 9: VoIP Delay for the Six Algorithms
The PLR for the 100 users, for each of the algorithms and for the three different flows are shown in Figures 6, 7 and 8. The lower the PLR value for each scheduler, the better the performance of the scheduler in terms of PLR. In figure 6, it is observed that FLS scheduler performed better than EXP rule, MLWDF, LOG rule, EXP-PF, and PF schedulers with an average difference of $8.2 \%, 17.9 \%$, $18.0 \%, 21.0 \%$, and $24.1 \%$ respectively. The reason is because target delay and queue size were used when the algorithm was developed.

Furthermore, the FLS scheduler performed better than the EXP rule, MLWDF, LOG rule, EXP-PF, and PF schedulers in terms of packet loss ratio as shown in Figure 7. The average data obtained from this graph show that the FLS scheduler has $3.1 \%, 5.8 \%, 5.9 \%, 6.4$ $\%$, and $12.1 \%$ better performance percentage over the EXP rule, MLWDF, LOG rule, EXP-PF, and PF schedulers respectively. It can also be observed that for this service, as the number of users grows the PLR increases for services.

Figure 8 shows the relationship between the PLR and the number of user for the BE flows. The FLS scheduler performed better than MLWDF, LOG rule, EXP rule, EXP$\mathrm{PF}$, and PF schedulers with an average data of $0.3 \%, 0.4$ $\%, 0.5 \%, 0.51 \%$, and $0.8 \%$ respectively.
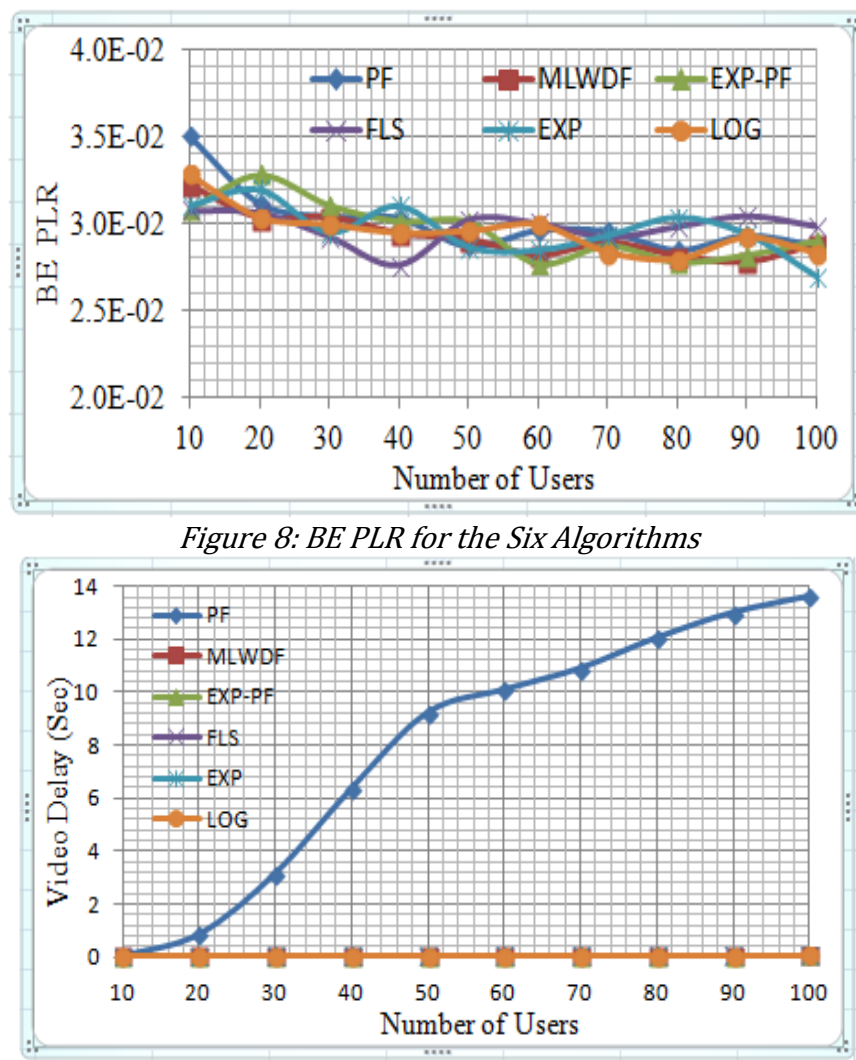

Figure 10: Video Delay for the Six Algorithms 
Generally, for all the flows considered, it is observed that VoIP flows experience considerably small PLR than video flows for all the six algorithms. This may be due to the fact that VoIP flows having a lower source bit rate gets higher priority from the scheduler.

Figure 9 shows the delay experienced by VoIP flows. As the number of users of the network increases, the delay increases for the six schedulers. However, the FLS scheduler performs better than the EXP-PF, LOG rule, MLWDF, EXP rule, and PF, schedulers with aggregate percentage values of $4.3 \%, 6.4 \%, 8.5 \%, 16.0 \%$, and 8.8 $\%$ respectively. Also, throughout the simulation, the packet delay of FLS gives lowest upper bound of the delay within the range of 0.01381 and $0.00859 \mathrm{sec}$.

The delay in term of Video flow experienced by the 100 users is shown in figure 10. The figure showed that FLS scheduler performed better than the EXP-PF, LOG rule, MLWDF, and EXP rule, and PF schedulers with aggregate percentage values of $0.13 \%, 0.131 \%, 0.15 \%$, and 0.154 $\%$, $95.89 \%$ respectively. This is because FLS computes the amount of data that each real-time source should transmit within a single frame, to satisfy its delay constrains.

The BE delay for different users is shown in figure 11 for the six schedulers. It is observed that the delay experienced by the BE packets is constantly at a low value of $1 \mathrm{msec}$ as shown in Figure 11 for the six

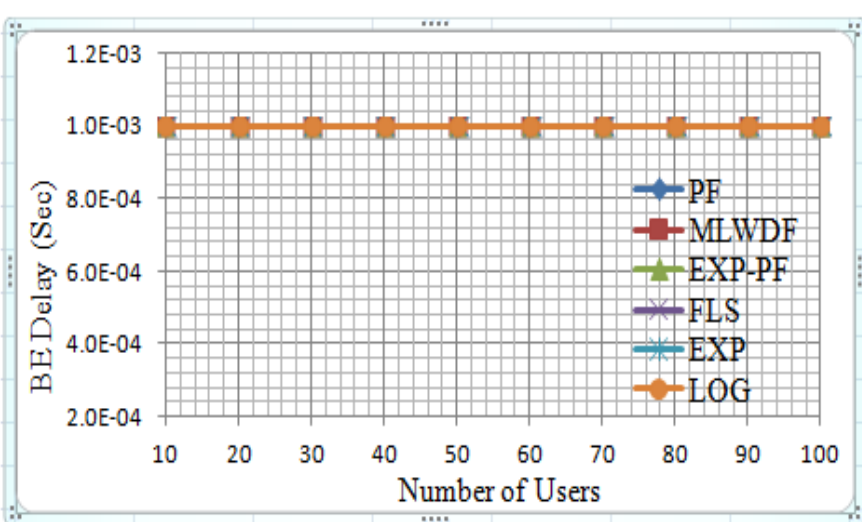

Figure 11: BE Delay for the Six Algorithms

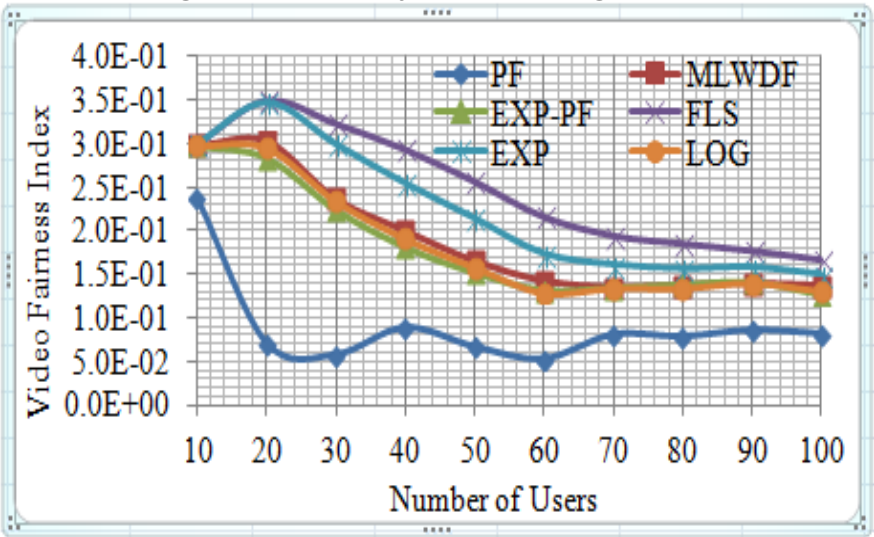

Figure 13: Video Fairness Index for the Six Algorithms schedulers. This is because, BE flows adopted a finite model.

The fairness index for each of the algorithms for the three different flows (VoIP, video and BE) are shown in figures 12, 13 and 14. Fairness index ranges from 0 to 1 . A system with a bigger fairness index is considered to be fairer. In figure 12, it is observed that the six algorithms showed closed fairness index. Also, the VoIP fairness index for the six algorithms decreases as the number of users was increasing.

However, the FLS scheduler performs better than the LOG rule, MLWDF, EXP-PF, EXP rule, and PF schedulers with aggregate percentage values of $0.025 \%, 0.068 \%$, $0.0812 \%, 0.97 \%$, and $0.118 \%$ respectively.

Figure 13 shows how fair each of the scheduler is to the Video flows. As the number of users of the network increases, the fairness index for the six schedulers decreases. However, the FLS scheduler performs better than the EXP rule, MLWDF, LOG rule, EXP-PF, PF, and schedulers with aggregate percentage values of $2.24 \%$, $5.10 \%, 5.53 \%, 5.91 \%$, and $13.96 \%$ respectively.

Figure 14 shows the level of fairness that was given to $\mathrm{BE}$ flows by the six schedulers. The average data collected inferred that, the MLWDF scheduler performed better than EXP-PF, LOG rule, EXP rule, PF, and FLS schedulers with an average difference of $0.05 \%, 0.051,0.06 \%, 0.21$ $\%$, and $11.32 \%$ respectively.

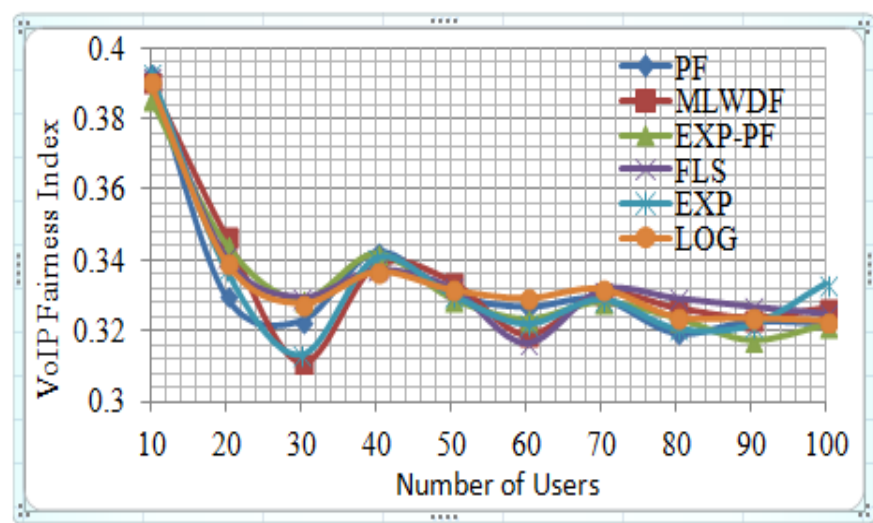

Figure 12: VoIP Fairness Index for the Six Algorithms

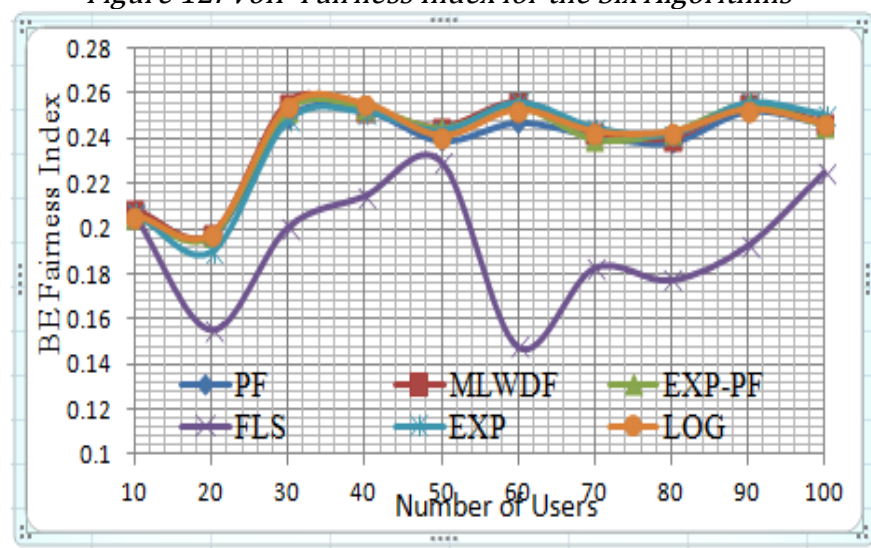

Figure 14: BE Fairness Index for the Six Algorithms 
Since MLWDF scheduler performed better while FLS scheduler performed least, it shows the FLS scheduler favored real-time services more than non-real-time services.

\section{CONCLUSION}

This paper presents a comparative analysis of the performance of six radio resource allocation algorithms in LTE networks. Our major focus was on the impact of each of the resource allocation algorithms on the VoIP, the Video, and the BE applications under overloaded conditions. In order to establish the impact of these resource allocation schemes on the QoS of the different applications in LTE networks, observations were made after the completion of the simulation and graphical analysis. We observed that;

- All the algorithms favor real-time services.

- The QoS experienced by the user is strongly dependent on the type of resource allocation algorithm deployed in eNB and the number of active users from a particular class of application.

- The FLS algorithm outperforms other algorithms in terms of delay, PLR, throughput, and fairness for VoIP and video flow. It was also observed that for BE flows, FLS outperforms other algorithms in terms of PLR but performed least in terms of throughput and fairness. This infers that increase in number of realtime applications users under overloaded condition will push the throughput of the non-real time users to the ground. This could be referred to as the starvation of non-real-time services.

We recommend that an efficient radio resource management method that can be fair to non-realtime services should be explored. At a very high network load therefore, certain kind of admission control and scheduling schemes are necessary if we want to give guaranteed service to real time traffic and some fairness of resource access to BE services. This action will prevent the starvation of best effort services at worst case scenarios. As part of future work, the researchers intend to use the concept of queueing theory based on Markov chain for QoS provision among multiclass applications.

\section{REFERENCES}

[1] Tokpo Ovengalt, C. B., Djouani, K., and Kurien, A., "A Fuzzy Approach for Call Admission Control in LTE Networks", 5th International Conference on Ambient Systems, Networks and Technologies, 237 - 244, 2014.

[2] ETSI TS 136213 V8.8.0; LTE; Evolved Universal Terrestrial Radio Access (E-UTRA); Physical layer procedure (3GPP TS 36.213 version 8.8.0 Release 8), European Telecommunication Standard Institute 2009.
[3] Nnamani, C. O., Anioke, C. L.,\& Ani, C. I., "Improved MLWDF Scheduler for LTE Downlink Transmission", International Journal of Electronics, 2016.

[4] Larmo, A., Lindström, M., Meyer, M., Pelletier, G., Torsner, J.,and Wiemann, H., "The LTE Link Layer Design (LTE part II: 3GPP Release 8)", IEEE Communication Magazine; pp. $52-59,2009$.

[5] Naik, P. U., Shirgeri, S., Udupi, G. R., Dias, P. F., "Design and Development of Medium Access Control Scheduler in LTE eNodeB", International Journal of Computational Engineering Research, Vol. 3, Issue 4, 2013.

[6] Li, X., Tam, K. W., and Fu, L., "Statistical Model of OFDM and its Application in Nonlinearity Analysis of LTE Advanced Systems", International Journal of Electronics Letters, 2015.

[7] Zyren, J., "Overview of 3GPP Long Term Evolution Physical Layer", Free Scale Semiconductor, 2007.

[8] Capozzi, F., Piro, G., Grieco, L. A., Boggia, G., and Camarda, P., "Downlink Packet Scheduling in LTE Cellular Networks: Key Design Issues and a Survey", IEEE Communications Surveys \& Tutorials, Vol. 15, No. 2, pp. 678 - 700, 2013.

[9] Ali-Yahiya, T, "Understanding LTE and its Performance Study of Opportunistic Scheduling in LTE Networks", Springer Science + Business Media, LLC, 2011.

[10] Zhu, C., Li, X., \& Fu, L., "Nonlinear Analysis of SC-FDMA Spectrum for LTE uplink", International Journal of Electronics Letters, vol. 2 issue 1, pp. 30-36, 2014.

[11] Sahoo, B., "Performance Comparison of Packet Scheduling Algorithms for Video Traffic in LTE Cellular Network", International Journal of Mobile Network Communication \& Telematics Vol. 3, No.3, 2013.

[12] Assaad M., \& Mourad A., "New Frequency-Time Scheduling Algorithms for 3GPP/LTE-like OFDMA Air Interface in the Downlink", IEEE Vehicular Technology Conference, 2008.

[13] Kwan, R., Leung, C., and Zhang, J., "Downlink Resource Scheduling in an LTE System", Mobile and Wireless Communications Physical Layer Development and Implementation, Salma Ait Fares and Fumiyuki (Ed.). InTech, pp. $190-207,2010$.

[14] Rhee, J. H., Holtzman, J. M., and Kim, D. K., "Performance Analysis of the Adaptive EXP/PF Channel Scheduler in an AMC/TDM system", IEEE Communication Letters, Vol. 8, pp. 4978-4980, 2004.

[15] Piro, G., Grieco, L. A., Boggia, G., Fortuna, R., and Camarda, P., "Two-Level Downlink Scheduling for Real-Time Multimedia Services in LTE Networks", IEEE Transaction on Multimedia, Vol. 13, No. 5, 2011.

[16] Omiyi, P. E., and Martini, M. G., "Cross-Layer Content/Channel Aware Multi-User Scheduling for Downlink Wireless Video Streaming", IEEE International Symposium on Wireless Pervasive Computing, pp. 412417, 2010.

[17] Ozcelebi, T., Oguz Sunay, M., Murat Tekalp A., and Reha Civanlar, M.,"Cross-Layer Optimized Rate Adaptation and Scheduling for Multiple User Wireless Video Streaming", IEEE Journal on Selected Areas in Communication Vol. 25, no. 4, pp. 760-769, 2007.

[18] Nonchev S., and Valkama, M., "Advanced Radio Resource Management for Multi-Antenna Packet Radio System", 
International Journal on Wireless \& Mobile Networks, Vol.2, No. 2, pp. 1-14, 2010.

[19] Singh, D., \& Singh, P., "Radio Resource Scheduling in 3GPP LTE: A Review", International Journal of Engineering Trends and Technology, Vol. 4, Issue 6; pp. 2405 - 2411, 2013.

[20] Fayssal, B., Abdennebi, M., \& Didi, F., "Survey on Scheduling and Radio Resources Allocation in LTE International Journal of Next-Generation Networks", Vol. 6, No.1, pp. 17 $29,2014$.

[21] WU, S., "A Channel Quality-aware Scheduling and Resource Allocation Strategy for Downlink LTE Systems", Journal of Computational Information Systems Vol. 8, No. 2, pp. 695707, 2012.

[22] Kela, P., Puttonen, J., Kolehmainen, N., Ristaniemi, T., Henttonen, T., and M. Moisio, "Dynamic Packet Scheduling Performance in UTRA Long Term Evolution Downlink", Proc. International Symposium on Wireless Pervasive Comput, Santorini, Greece, pp. 308 - 313, 2008.

[23] Tanenbaum, A. S., “Modern Operating Systems”, Upper Saddle River, NJ, USA Prentice Hall Press, 2007.

[24] Liu D., and Lee, Y.H., "An Efficient Scheduling Discipline for Packet Networks using Earliest Deadline First Round Robin", Proc. IEEE Int. Conf. on Computer Commun. and Net., ICCCN, Dallas, USA, pp. 5 - 1, 2003.

[25] Lin Y., and Yue, G., "Channel-Adapted and Buffer-Aware Packet Scheduling in LTE Wireless Communication System", Proc. IEEE Wireless Commun., Net. and Mobile Comput., WiCOM, Dalian, China, pp. 1-4, 2008.

[26] Holtzman, J. M., “Asymptotic Analysis of Proportional Fair Algorithm", IEEE International Symposium on Personal, Indoor and Mobile Radio Communications Vol. 2, pp. 33, 2001.

[27] Kushner, H. J., \& Whiting, P. A., "Convergence of Proportional-Fair Sharing Algorithms under General Conditions", IEEE Transactions on Wireless Communications, 3(4), pp. 1250-1259, 2004.

[28] Pokhariyal, A., Kolding, T. E., and Mogensen, P. E., "Performance of Downlink Frequency Domain Packet Scheduling for the UTRAN Long Term Evolution" IEEE 17th international symposium on personal, indoor and mobile radio communications pp. 15, 2006.

[29] Pokhariyal, A., Monghal, G., Pedersen, K., Mogensen, P., Kovacs, I., Rosa, C., \& Kolding, T., "Frequency Domain Packet Scheduling Under Fractional Load for the UTRAN LTE Downlink. IEEE 65th Vehicular Technology Conference, 2007, VTC2007-Spring pp. 699-703, 2007.

[30] Kolding, T. E., "Link and System Performance Aspects of Proportional Fair Scheduling in WCDMA/HSDPA. IEEE 58th Vehicular Technology Conference, 2003. VTC 2003 Fall Vol. 3, pp. 1717-1722, 2003.

[31] Wang, B., Pedersen, K. I., Kolding, T. E., and Mogensen, P. E., "Performance of VoIP on HSDPA", IEEE 61st Vehicular Technology Conference, 2005. VTC 2005-Spring Vol. 4, pp. 2335-2339, 2005.
[32] Kwan, R., Leung,C., and Zhang, J., "Multi user scheduling on the downlink of an LTE cellular system. Research Letters in Communications, 2008, 3, 2008.

[33] Wei, N., Pokhariyal, A., Sorensen, T., Kolding, T., and Mogensen, P., Performance of MIMO with Frequency Domain Packet Scheduling in UTRAN LTE downlink. IEEE 65th Vehicular Technology Conference, 2007. VTC2007Spring pp. 1177-1181, 2007.

[34] Xiaoxin, Q., and Kapil, C., "On the performance of adaptive modulation in cellular systems, IEEE Transactions on Communications, 47(6), 884-895, 1999.

[35] Jalali, A., Padovani, R., and Pankaj, R., "Data Throughput of CDMAHDR a High Efficiency-High Data Rate Personal Communication Wireless System", IEEE 51st Vehicular Technology Conference Proceedings, Tokyo, pp. 18541858, 2000.

[36] Andrews, M., Kumaran, K., Ramanan, K., Stolyar, A., Vijayakumar, R., and Whiting, P. "Providing Quality of Service over a Shared Wireless Link" IEEE Communications Magazine, vol. 39, pp. 150-154, 2001.

[37] Sulthana, S. F., and Nakkeeran, R., Study of Downlink Scheduling Algorithms in LTE Networks, Journal of Networks, Vol., 9, No. 12, 2014.

[38] Basukala, R., Mohd Ramli, H. A , and S. and rasegaran, K., "Performance Analysis of EXP/PF and M-LWDF in Downlink 3GPP LTE system", IEEE, 2009.

[39] Shakkottai S., and Stolyar, A., "Scheduling for Multiple Flows Sharing a Time-Varying Channel: The Exponential Rule, Analytical Methods in Applied Probability", American Mathematical Society Translations, Vol. 207, Series 2, pp. 185-202, 2002.

[40] Singh, D. and Singh, P., "Radio Resource Scheduling in 3GPP LTE: A Review", International Journal of Engineering Trends and Technology, Vol. 4, Issue 6, 2013.

[41]Sadiq, B., baek S. J., and Vaciana, G., "Delay Opitmal Opportunistic Scheduling and Approximations: The Log Rule", Proceedings of the 27th Annual Joint Conference on the IEEE Computer and Communication Societies pp. 19, 2009.

[42] Biernacki, A., Tutschku, K., "Comparative Performance Study of LTE Downlink Schedulers", Wireless Pers Commun 74: pp. 585-599, 2014.

[43] Kumar A., Garg A. K., "Queuing Algorithm Based Quality of Service (QoS) for Scheduling Environment Model in WIMAX Network with Opnet Modeler", Global Journal of Research in Engineering Electronic and Electronic Engineering, Vol. 11 Issue 8 Version 1.0, 2011

[44] https://en.m.wikipedia.org/wiki/LTE-Sim

[45] Piro, G., Grieco, L. A., Boggia, G., Capozzi F., and Camarda, P., "Simulating LTE Cellular Systems: An Open-Source Framework", IEEE Transaction on Vehicular Technology, Vol. 60, No. 2, 2011.

[46] Wang, L., "Resource Allocation in OFDMA Relay Enhanced Cellular Networks", Doctoral thesis, 2010. 